\title{
Effect of Tillage and Weed management practices on Weed Control and Yield in Wheat
}

\author{
S. Pratikshya Rani ${ }^{1 *}$, B. Duary ${ }^{1}$ and Sidhartha Priyatam ${ }^{2}$ \\ ${ }^{1}$ Department of Agronomy, Palli Siksha Bhavana, Visva-Bharati, West Bengal, India, \\ ${ }^{2}$ College of Postgraduate Studies in Agricultural Sciences, (CAU-Imphal), \\ Umiam-793103, Meghalaya, India \\ *Corresponding author
}

\section{A B S T R A C T}

\begin{tabular}{l} 
Ke y w o r d s \\
Zero tillage, \\
Pendimethalin, \\
Straw mulching, \\
Tillage, Wheat, \\
Weed management \\
\hline Article Info \\
\hline $\begin{array}{l}\text { Accepted: } \\
\text { 18 May 2020 } \\
\text { Available Online: } \\
\text { 10 June } 2020\end{array}$ \\
\hline
\end{tabular}

\section{Introduction}

Wheat (Triticum aestivum L.) being the most valuable crop for vast global population is essential for food security of a country.
A field experiment was conducted during the rabi season of 2017-18 at the Agricultural Farm of the Institute of Agriculture (PalliSikshaBhavana), Visva-Bharati, Sriniketan, West Bengal to study the effect of tillage and weed management practices on weed population dynamics and growth in wheat. The experiment was laid out in split-plot design with three replications. Two tillage practices comprising of Zero tillage (ZT) and Conventional tillage (CT) and eight weed management practices viz. Straw mulching alone at $4.0 \mathrm{t} \mathrm{ha}^{-1}$, Pendimethalin at $0.75 \mathrm{~kg} \mathrm{ha}^{-1}$, Clodinafoppropargyl 15\% + Metsulfuron methyl 1\% WP at $0.40 \mathrm{~kg} \mathrm{ha-}{ }^{1}$, Pendimethalin at $0.75 \mathrm{~kg} \mathrm{ha}^{-1}$ straw mulching at $4.0 \mathrm{t} \mathrm{ha}^{-1}$, Pendimethalin at $0.75 \mathrm{~kg} \mathrm{ha}^{-1} \mathrm{fbclodinafoppropargyl} 15 \%+$ metsulfuron methyl $1 \% \mathrm{WP}$ at $0.40 \mathrm{~kg}$ at, Straw mulching alone at $4.0 \mathrm{t} \mathrm{ha}^{-1} \mathrm{fbclodinafoppropargyl} 15 \%+$ metsulfuron methyl $1 \% \mathrm{WP}$ at $0.40 \mathrm{~kg}$, Weed free and Weedy check were assigned to the sub-plot. It was witnessed that ZT caused a substantial reduction in the population of narrow-leaved and broad-leaved compared to CT. Among the weed management practicespendimethalin at $0.75 \mathrm{~kg} \mathrm{ha}^{-1} \mathrm{fb}$ straw mulching at $4.0 \mathrm{t} \mathrm{ha}^{-1}$, pendimethalin at $0.75 \mathrm{~kg} \mathrm{ha}^{-1} \mathrm{fb}$ clodinafop-propargyl + metsulfuron methyl $0.4 \mathrm{~kg} \mathrm{ha}^{-1}$ and sole application of pendimethalin at $0.75 \mathrm{~kg} \mathrm{ha} \mathrm{ha}^{-1}$ registered the lowest density as well as biomass of grassy weeds while straw mulching $f b$ clodinafop-propargyl + MSM at $0.40 \mathrm{~kg} \mathrm{ha}^{-1}$ and pendimethalin at $0.75 \mathrm{~kg} \mathrm{ha}^{-1} \mathrm{fb}$ clodinafop-propargyl + metsulfuron methyl $0.4 \mathrm{~kg} \mathrm{ha}^{-1}$ recorded the lowest density as well as biomass broadleaved weeds. Lower values of weed density, total weed biomass and higher yield were registered with combination of zero tillage with integrated use of preemergence herbicide pendimethalin at $0.75 \mathrm{~kg} \mathrm{ha}^{-1}$ with straw mulching at $4.0 \mathrm{t} \mathrm{ha} \mathrm{ha}^{-1}$ and pendimethalin at $0.75 \mathrm{~kg} \mathrm{ha}^{-1} f b$ clodinafop-propargyl + metsulfuron methyl at $0.4 \mathrm{~kg} \mathrm{ha}$. 
spinosus, Gnaphalium indicum, Spilanthes calva and Cyperus rotundus became predominant weed flora in wheat (Mondal and Duary, 2009; Rahaman and Mukherjee, 2009; Pawar et al., 2017 and Rana et al., 2017).

Conventional tillage through tractor not only results in soil loss but also higher weed seed germination. While, zero tillage mainly needs to build up a defensive cover of residues on soil surface. Zero tillage, largely relying on deposition of crop residues requires appropriate method for placement of seeds on the upper soil layer. As a result of which, relative spread of unwanted weed flora can be minimized to a great extent (Susha et al., 2014). Zero tillage is a great option now a days which is enjoying immense response from Indian farmers as it increases both productivity and sustainability. ZT wheat results in higher weed control efficiency over CT by decreasing weed density and dry matter considerably at Varanasi (Prasad et al., 2005).

Mulching plays a pivotal role in yield enhancement through increasing water holding capacity of soil and by checking weed population. Application of residue mulch in ZT wheat considerably reduced weed flora over time than CT wheat (Kumar et al., 2015).

In situations of mixed weed flora, pre- mix or consecutive application of herbicides with varied selectivity not only facilitates in efficient weed management but also results in minimizing cost and time, and improving biological activity than their discrete applications (Sharma et al., 2015). Tank-mix or pre-mix use of different herbicide chemistries or sequential application of preand post-emergence herbicides at different times showed effective weed control (Baghestani et al., 2008). Besides managing mixed weed flora, the integrated use of herbicides may help in managing herbicide resistance problems. So, keeping all these things in mind this experiment was carried out to find out the effect of integrated use of herbicide and straw mulching under different tillage practices to control the weed flora in wheat.

\section{Materials and Methods}

The field experiment entitled "Weed management in wheat by integrated use of herbicide and straw mulch under different tillage practices" was conducted in the Agricultural Farm of Palli Siksha Bhavana (Institute of Agriculture), Visva-Bharati, Sriniketan, Birbhum, West Bengal duringrabi season of 2017-18. The soil of the experimental field was sandy loam (Ultisol), having $\mathrm{pH}$ 5.6, organic carbon 0.38, available $\mathrm{N}, \mathrm{P}$ and $\mathrm{K} 138.2 \mathrm{~kg}, 25.1 \mathrm{~kg}$ and $120.41 \mathrm{~kg}$, respectively. The field experiment was conducted in split plot design with three replication. The treatments included two different tillage practices in main plots viz. Zero Tillage; and Conventional Tillage and eight weed management practices in subplots. viz. Straw mulching alone at $4.0 \mathrm{tha}^{-1}$ at 20 DAS, Pendimethalin at $0.75 \mathrm{~kg} \mathrm{ha}^{-1}$ at 1 DAS, Clodinafoppropargyl $15 \%+$ metsulfuron methyl $1 \% \mathrm{WP}$ at $0.40 \mathrm{~kg} \mathrm{ha}^{-1}$ at 35 DAS, Pendimethalin at $0.75 \mathrm{~kg} \mathrm{ha}^{-1}$ at 1DAS $f b$ straw mulching at $4.0 \mathrm{tha}^{-\mathrm{T}} 20$ DAS, Pendimethalin at $0.75 \mathrm{~kg} \mathrm{ha}^{-1}$ at 1 DAS followed by clodinafoppropargyl $15 \%$ +metsulfuron methyl $1 \% \mathrm{WP}$ at $0.40 \mathrm{~kg}$ at 35 DAS, Straw mulching alone at $4.0 \mathrm{t} \mathrm{ha}^{-1}$ at 20 DAS $\mathrm{fb}$ clodinafoppropargyl $15 \%+$ metsulfuron methyl $1 \% \mathrm{WP}$ at $0.40 \mathrm{~kg}$ at 35 DAS, Weed free and Weedy Check.

The conventional tillage plots were ploughed twice by tractor drawn plough and finally planking was done to have a uniform seed bed of fine tilth. No tillage operation was carried out in zero tillage plots. The wheat variety selected for the experiment was HD 2824 
(Poorva). In case of conventional tillage line sowing of wheat was followed with a row to row spacing of $20 \mathrm{~cm}$. In case of zero tillage wheat was mechanically sown with national zero till ferti-seed drill machine. Half quantity of nitrogen and full amount of phosphorus and potassium were applied in each plot as basal on the day of sowing. Remaining half quantity of $\mathrm{N}$ was applied in two equal splits as top dressing. The first irrigation was given at crown root initiation stage (22 DAS). Afterwards four irrigations were given at tillering, jointing, flowering and at milking stage. The crop was harvested by sickles manually at physiological maturity. The bundles were properly tagged, and sun dried before threshing on the concrete floor and plot-wise weight of grains and straws were recorded separately.

\section{Results and Discussion}

The prominent weed species witnessed in the experimental site were Digitaria sanguinalis, among the grasses and Gnaphalium indicum, Polygonum plebeium and Spilanthes calva among broadleaved weeds.

\section{Effect on weeds}

At both 45DAS and 75DAS, the density of grassy weeds continues to be more in conventional tillage than zero tillage. At 75DAS, significantly lowest weed density was found in ZT than CT (Table 1). The effect of weed management practices on density of grasses was significant. The treatment pre-emergence application of pendimethalin at $0.75 \mathrm{~kg} \mathrm{ha}^{-1}\left(\mathrm{~W}_{2}\right)$, pendimethalin at $0.75 \mathrm{~kg} \mathrm{ha}^{-1} \mathrm{fb}$ straw mulching at $4.0 \mathrm{t} \mathrm{ha}^{-1}$ at $20 \mathrm{DAS}\left(\mathrm{W}_{4}\right)$ and pendimethalin at $0.75 \mathrm{~kg} \mathrm{ha}^{-1} \mathrm{fb}$ clodinafoppropargyl + metsulfuron methyl (MSM) at 0.4 $\mathrm{kg} \mathrm{ha}^{-1}\left(\mathrm{~W}_{5}\right)$ effectively controlled grassy weeds and listed zero count in both 45DAS and 75DAS. These treatments were superior to remaining treatments in controlling grassy weeds and even comparable with weed free check $\left(\mathrm{W}_{7}\right)$ also.

The density of broad leaved weeds was found significantly higher in conventional tillage than zero tillage at both 45 and 75 DAS (Table 1).Pendimethalin at $0.75 \mathrm{~kg} \mathrm{ha}^{-1} \mathrm{fb}$ clodinafop-propargyl + MSM at $0.4 \mathrm{~kg} \mathrm{ha}^{1}$ $\left(\mathrm{W}_{5}\right)$ was found to be most effective in controlling broadleaved weeds and the treatment was at par with weed free check registering zero count of broadleaved weed at 45DAS. While, clodinafop-propargyl + metsulfuron methyl at $0.4 \mathrm{~kg} \mathrm{ha}\left(\mathrm{W}_{3}\right)$, integrated use of pendimethalin at $0.75 \mathrm{~kg} \mathrm{ha}^{-}$ $1 \mathrm{fb}$ clodinafop-propargyl + metsulfuron methyl at $0.4 \mathrm{~kg} \mathrm{ha}^{1}\left(\mathrm{~W}_{5}\right)$ and straw mulching $f b$ clodinafop-propargyl + MSM at $0.40 \mathrm{~kg} \mathrm{ha}^{-}$ ${ }^{1}\left(\mathrm{~W}_{6}\right)$ listed zero count of broadleaved weeds at 75 DAS and were statistically at par with weed free check $\left(\mathrm{W}_{7}\right)$.

The dry weight of grassy weed was higher in conventional tillage. All the weed management practices brought significant reduction in the biomass of grassy weed over weedy check $\left(\mathrm{W}_{8}\right)($ Table 2$)$. The treatment pre-emergence application of pendimethalin at $0.75 \mathrm{~kg} \mathrm{ha}^{1}\left(\mathrm{~W}_{2}\right)$, pendimethalin at $0.75 \mathrm{~kg}$ $\mathrm{ha}^{-1} \mathrm{fb}$ straw mulching at $4.0 \mathrm{t} \mathrm{ha}^{-1}$ at $20 \mathrm{DAS}$ $\left(\mathrm{W}_{4}\right)$ and pendimethalin at $0.75 \mathrm{~kg} \mathrm{ha}^{-1} \mathrm{fb}$ clodinafop-propargyl + metsulfuron methyl at $0.4 \mathrm{~kg}$ ha ${ }^{1}\left(\mathrm{~W}_{5}\right)$ registered no dry weight at both 45 and 75 DAS. These treatments were even comparable with weed free check $\left(\mathrm{W}_{7}\right)$, also reducing the biomass of grasses by $100 \%$.

Biomass of broad leaved weeds was found significantly higher in conventional tillage $\left(\mathrm{T}_{2}\right)$ over zero tillage $\left(\mathrm{T}_{1}\right)$ at both 45 and 75DAS. Pendimethalin at $0.75 \mathrm{~kg} \mathrm{ha}^{-1} \mathrm{fb}$ clodinafop-propargyl + metsulfuron methyl $0.4 \mathrm{~kg} \mathrm{ha}^{1}\left(\mathrm{~W}_{5}\right)$ completely controlled broad leaved weeds and registered no dry weight at 45DAS.While, sole application of clodinafoppropargyl + MSM at $0.40 \mathrm{~kg} \mathrm{ha}^{-1}\left(\mathrm{~W}_{3}\right)$, 
integrated use of pendimethalin at $0.75 \mathrm{~kg}$ $\mathrm{ha}^{-1} f b$ clodinafop-propargyl + metsulfuron methyl at $0.4 \mathrm{~kg} \mathrm{ha}^{1}\left(\mathrm{~W}_{5}\right)$ and straw mulching $f b$ clodinafop-propargyl + MSM at $0.40 \mathrm{~kg}$ $\mathrm{ha}^{-1}\left(\mathrm{~W}_{6}\right)$ registered the lowest biomass and was statistically at par with weed free $\left(\mathrm{W}_{7}\right)$ at 75DAS.

Among integrated weed management practices pendimethalin at $0.75 \mathrm{~kg} \mathrm{ha}^{-1} \mathrm{fb}$ clodinafop-propargyl + metsulfuron methyl $0.4 \mathrm{~kg} \mathrm{ha}^{1}\left(\mathrm{~W}_{5}\right)$ recorded the highest WCE $(100 \%)$ which was followed by pendimethalin at $0.75 \mathrm{~kg} \mathrm{ha}^{-1} \mathrm{fb}$ straw mulching at $4.0 \mathrm{t} \mathrm{ha}^{-1}$ $\left(\mathrm{W}_{4}\right)$ and straw mulching $f b$ clodinafop- propargyl + MSM at $0.40 \mathrm{~kg} \mathrm{ha}^{-1}\left(\mathrm{~W}_{6}\right)($ Table $3)$.

The combined use of pendimethalin along with straw mulching or post emergence herbicide displayed better weed control efficiency because of the explanations that component method was used in different time in the growing period thus covering the whole spectrum of weeds and keeping the weed pressure below throughout the period. In addition to herbicide, application of straw mulch suppressed the weeds, which emerged during later stage of crop growth.

Table.1 Effect of tillage and weed management practices on weed density at 45 DAS and 75 DAS in wheat

\begin{tabular}{|c|c|c|c|c|}
\hline Treatments & 45 DAS & & 75 DAS & \\
\hline Tillage practices & Grass & BLW & Grass & BLW \\
\hline$T_{1}-$ Zero tillage & $5.86(33.90)$ & $8.22(67.01)$ & $6.28(38.89)$ & $9.20(84.12)$ \\
\hline $\mathrm{T}_{2}$ - Conventional tillage & $6.12(36.97)$ & $9.43(88.37)$ & $6.58(42.80)$ & $10.03(100.15)$ \\
\hline S.Em $( \pm)$ & 0.11 & 0.03 & 0.04 & 0.03 \\
\hline $\operatorname{LSD}(\mathrm{P}=0.05)$ & NS & 0.18 & 0.27 & 0.16 \\
\hline \multicolumn{5}{|l|}{ Weed management practices } \\
\hline $\begin{array}{l}\mathrm{W}_{1} \text { - Straw mulching at } 4.0 \mathrm{tha}^{-1} \text { at } 20 \\
\text { DAS }\end{array}$ & 10.08(101.06) & $\begin{array}{c}13.94 \\
(193.81)\end{array}$ & $\begin{array}{c}10.12 \\
(101.90)\end{array}$ & $18.76(351.26)$ \\
\hline $\mathrm{W}_{2^{-}}$Pendimethalin at $0.75 \mathrm{~kg} \mathrm{ha}^{-1}$ & $0.71(0.00)$ & $8.79(76.69)$ & $0.71(0.00)$ & $18.58(344.67)$ \\
\hline $\begin{array}{l}\mathrm{W}_{3} \text {-Clodinafop-propargyl }+ \text { MSM at } \\
0.40 \\
\quad \mathrm{~kg} \mathrm{ha}^{-1} \text { at } 35 \text { DAS }\end{array}$ & $12.22(148.84)$ & $\begin{array}{c}10.88 \\
(117.85)\end{array}$ & $\begin{array}{c}14.52 \\
(210.40)\end{array}$ & $0.71(0.00)$ \\
\hline $\begin{array}{l}\mathrm{W}_{4}-\mathrm{W}_{2} f b \text { straw mulching at } 4.0 \mathrm{tha}^{-1} \text { at } \\
20 \text { DAS }\end{array}$ & $0.71(0.00)$ & $8.10(65.18)$ & $0.71(0.00)$ & $11.98(142.95)$ \\
\hline $\begin{array}{l}\mathrm{W}_{5}-\mathrm{W}_{2} f b \text { clodinafop-propargyl + MSM } \\
0.4 \mathrm{~kg} \mathrm{ha}^{-1} 35 \text { DAS }\end{array}$ & $0.71(0.00)$ & $0.71(0.00)$ & $0.71(0.00)$ & $0.71(0.00)$ \\
\hline $\begin{array}{l}\mathrm{W}_{6}-\mathrm{W}_{1} f b \text { clodinafop-propargyl }+\mathrm{MSM} \\
\text { at } 0.40 \mathrm{~kg} \mathrm{ha}^{-1} \text { at } 35 \mathrm{DAS}\end{array}$ & $8.68(74.84)$ & $8.40(70.10)$ & 7.76(59.68) & $0.71(0.00)$ \\
\hline $\mathrm{W}_{7}$-Weed free & $0.71(0.00)$ & $0.71(0.00)$ & $0.71(0.00)$ & $0.71(0.00)$ \\
\hline $\mathrm{W}_{8}$-Weedy check & 14.14(199.39) & $\begin{array}{c}19.05 \\
(362.37)\end{array}$ & $\begin{array}{c}16.20 \\
(261.90)\end{array}$ & $24.78(613.77)$ \\
\hline S.Em $( \pm)$ & 0.33 & 0.35 & 0.17 & 0.21 \\
\hline $\operatorname{LSD}(\mathrm{P}=0.05)$ & 0.97 & 1.02 & 0.49 & 0.60 \\
\hline
\end{tabular}


Table.2 Effect of tillage and weed management practices on weed density at 45 DAS and 75 DAS in wheat

\begin{tabular}{|c|c|c|c|c|}
\hline Treatments & 45 DAS & & 75 DAS & \\
\hline Tillage practices & Grass & BLW & Grass & BLW \\
\hline $\mathrm{T}_{1}$ - Zero tillage & $1.67(2.30)$ & $1.81(2.79)$ & $2.31(4.85)$ & $3.14(9.36)$ \\
\hline $\mathrm{T}_{2}$ - Conventional tillage & $1.85(2.93)$ & $2.25(4.56)$ & $2.48(5.67)$ & $3.04(8.770$ \\
\hline S.Em $( \pm)$ & 0.03 & 0.04 & 0.02 & 0.01 \\
\hline $\operatorname{LSD}(\mathrm{P}=0.05)$ & NS & 0.22 & 0.15 & 0.07 \\
\hline \multicolumn{5}{|l|}{ Weed management practices } \\
\hline $\mathrm{W}_{1}$ - Straw mulching at $4.0 \mathrm{tha}^{-1}$ at $20 \mathrm{DAS}$ & $2.95(8.21)$ & $2.85(7.62)$ & $3.39(10.96)$ & $5.36(28.22)$ \\
\hline $\mathrm{W}_{2}$ - Pendimethalin at $0.75 \mathrm{~kg} \mathrm{ha}^{-1}$ & $0.71(0.00)$ & $1.82(2.83)$ & $0.71(0.00)$ & $5.33(27.88)$ \\
\hline $\begin{array}{l}\mathrm{W}_{3} \text {-Clodinafop-propargyl + MSM at } 0.40 \\
\mathrm{~kg} \mathrm{ha}^{-1} \text { at } 35 \text { DAS }\end{array}$ & $3.01(8.59)$ & $2.32(4.89)$ & $4.73(21.91)$ & $0.71(0.00)$ \\
\hline $\begin{array}{l}\mathrm{W}_{4}-\mathrm{W}_{2} f b \text { straw mulching at } 4.0 \mathrm{tha}^{-1} \text { at } 20 \\
\text { DAS }\end{array}$ & $0.71(0.00)$ & $1.95(3.30)$ & $0.71(0.00)$ & $3.41(11.11)$ \\
\hline $\begin{array}{l}\mathrm{W}_{5^{-}}-\mathrm{W}_{2} f b \text { clodinafop-propargyl + MSM } \\
0.4 \mathrm{~kg} \mathrm{ha}^{-1} 35 \text { DAS }\end{array}$ & $0.71(0.00)$ & $0.71(0.00)$ & $0.71(0.00)$ & $0.71(0.00)$ \\
\hline $\begin{array}{l}\mathrm{W}_{6}-\mathrm{W}_{1} f b \text { clodinafop-propargyl }+ \text { MSM at } \\
0.40 \mathrm{~kg} \mathrm{ha}^{-1} \text { at } 35 \text { DAS }\end{array}$ & $2.12(4.00)$ & 1.87(2.99) & $2.71(6.86)$ & $0.71(0.00)$ \\
\hline $\mathrm{W}_{7}$-Weed free & $0.71(0.00)$ & $0.71(0.00)$ & $0.71(0.00)$ & $0.71(0.00)$ \\
\hline $\mathrm{W}_{8}$-Weedy check & $3.18(9.64)$ & $4.02(15.69)$ & $5.52(30.00)$ & 7.81(60.57) \\
\hline S.Em $( \pm)$ & 0.12 & 0.12 & 0.10 & 0.09 \\
\hline $\operatorname{LSD}(\mathrm{P}=0.05)$ & 0.35 & 0.34 & 0.30 & 0.26 \\
\hline
\end{tabular}

Table.3 Effect of weed management practices on weed control efficiency in wheat

\begin{tabular}{|c|c|c|}
\hline \multirow[t]{2}{*}{ Treatments } & \multicolumn{2}{|c|}{$\begin{array}{c}\text { Weed Control Efficiency } \\
(\%)\end{array}$} \\
\hline & 45 DAS & 75 DAS \\
\hline \multicolumn{3}{|l|}{ Weed management practices } \\
\hline $\mathrm{W}_{1}$ - Straw mulching at $4.0 \mathrm{t} \mathrm{ha}^{-1}$ at $20 \mathrm{DAS}$ & 36.89 & 56.74 \\
\hline $\mathrm{W}_{2}$ - Pendimethalin at $0.75 \mathrm{~kg} \mathrm{ha}^{-1}$ & 88.85 & 69.23 \\
\hline $\begin{array}{l}\mathrm{W}_{3} \text {-Clodinafop-propargyl + MSM at } 0.40 \\
\mathrm{~kg} \mathrm{ha}^{-1} \text { at } 35 \text { DAS }\end{array}$ & 46.68 & 75.82 \\
\hline $\begin{array}{l}\mathrm{W}_{4}-\mathrm{W}_{2} f b \text { straw mulching at } 4.0 \mathrm{tha}^{-1} \text { at } 20 \\
\text { DAS }\end{array}$ & 86.99 & 87.74 \\
\hline $\begin{array}{l}\mathrm{W}_{5}-\mathrm{W}_{2} f b \text { clodinafop-propargyl + MSM } \\
0.4 \mathrm{~kg} \mathrm{ha}^{-1} 35 \text { DAS }\end{array}$ & 100.00 & 100.00 \\
\hline $\begin{array}{l}\mathrm{W}_{6}-\mathrm{W}_{1} f b \text { clodinafop-propargyl }+\mathrm{MSM} \text { at } 0.40 \\
\mathrm{~kg} \mathrm{ha}^{-1} \text { at } 35 \text { DAS }\end{array}$ & 72.18 & 92.43 \\
\hline $\mathrm{W}_{7}$-Weed free & 100.00 & 100.00 \\
\hline $\mathrm{W}_{8}$-Weedy check & 0.00 & 0.00 \\
\hline
\end{tabular}


Table.4 Effect of tillage and weed management practices on earsm ${ }^{-2}$, grain yield $\left(\mathrm{kg} \mathrm{ha}^{-1}\right)$, straw yield $\left(\mathrm{kg} \mathrm{ha}^{-1}\right)$ and return rupee ${ }^{-1}$ invested of wheat

\begin{tabular}{|c|c|c|c|c|}
\hline Treatment & $\begin{array}{l}\text { Ears } \\
\mathbf{m}^{-2}\end{array}$ & $\begin{array}{c}\text { Grain yield } \\
\left(\mathrm{kg} \mathrm{ha}^{-1}\right)\end{array}$ & $\begin{array}{c}\text { Straw } \\
\text { yield } \\
\left(\mathrm{kg} \mathrm{ha}^{-1}\right)\end{array}$ & $\begin{array}{c}\text { Return } \\
\text { rupee }{ }^{-1} \\
\text { invested }\end{array}$ \\
\hline \multicolumn{5}{|l|}{ Tillage practices } \\
\hline $\mathrm{T}_{1}$-Zero tillage & 258.5 & 3124 & 4506 & 2.06 \\
\hline $\mathrm{T}_{2}$-Conventional tillage & 251.4 & 2845 & 4165 & 1.45 \\
\hline $\operatorname{S.Em~}( \pm)$ & 3.36 & 71.92 & 90.68 & 0.04 \\
\hline $\operatorname{LSD}(\mathrm{P}=0.05)$ & NS & NS & NS & 0.26 \\
\hline \multicolumn{5}{|l|}{ Weed management practices } \\
\hline $\begin{array}{l}\mathrm{W}_{1^{-}} \text {Straw mulching at } 4.0 \mathrm{tha}^{-1} \text { at } 20 \\
\text { DAS }\end{array}$ & 252.4 & 2659 & 4028 & 1.51 \\
\hline $\mathrm{W}_{2^{-}}$Pendimethalin at $0.75 \mathrm{~kg} \mathrm{ha}^{-1}$ & 223.6 & 3011 & 4464 & 2.03 \\
\hline $\begin{array}{l}\mathrm{W}_{3} \text { - Clodinafop-propargyl + MSM at } \\
0.40 \mathrm{~kg} \mathrm{ha}^{-1} \text { at } 35 \mathrm{DAS}\end{array}$ & 232.7 & 2669 & 3871 & 1.77 \\
\hline $\begin{array}{l}\mathrm{W}_{4}-\mathrm{W}_{2} f b \text { straw mulching at } 4.0 \mathrm{t} \mathrm{ha}^{-1} \text { at } 20 \\
\text { DAS }\end{array}$ & 270.0 & 3486 & 4735 & 1.87 \\
\hline $\begin{array}{l}\mathrm{W}_{5^{-}} \mathrm{W}_{2} f b \text { clodinafop-propargyl + MSM } \\
0.4 \mathrm{~kg} \mathrm{ha}^{-1} 35 \text { DAS }\end{array}$ & 265.8 & 3283 & 4666 & 2.07 \\
\hline $\begin{array}{l}\mathrm{W}_{6}-\mathrm{W}_{1} f b \text { clodinafop-propargyl }+\mathrm{MSM} \text { at } \\
0.40 \mathrm{~kg} \mathrm{ha}^{-1} \text { at } 35 \mathrm{DAS}\end{array}$ & 289.7 & 3327 & 4987 & 1.81 \\
\hline $\mathrm{W}_{7}$-Weed free & 282.8 & 3504 & 4784 & 1.57 \\
\hline $\mathrm{W}_{8}$-Weedy check & 222.5 & 1939 & 3148 & 1.41 \\
\hline S.Em $( \pm)$ & 9.72 & 139.62 & 168.93 & 0.07 \\
\hline $\operatorname{LSD}(\mathrm{P}=0.05)$ & 28.16 & 404.41 & 489.30 & 0.20 \\
\hline
\end{tabular}

Pendimethalin managed the weeds which appeared in wheat at initial stage of crop growth. The weeds appeared subsequently were again controlled by component method. Number of workers put forwarded opinions regarding beneficial effect of integrated weed management practices over unweeded control (Singh et al., 2005).

\section{Effect on crop}

Although zero tillage recorded higher number of ears than conventional tillage but no significant difference was found among the tillage practices. However, significant effect of weed management practices was observed on number of ears. Straw mulching $f b$ clodinafop-propargyl + MSM at $0.40 \mathrm{~kg} \mathrm{ha}^{-}$ ${ }^{1}\left(\mathrm{~W}_{6}\right)$ reported significantly higher number of ears which was statistically at par with weed free $\left(\mathrm{W}_{7}\right)$, pendimethalin at $0.75 \mathrm{~kg} \mathrm{ha}^{-1} \mathrm{fb}$ straw mulching at $4.0 \mathrm{t} \mathrm{ha}^{-1}\left(\mathrm{~W}_{4}\right)$ and pendimethalin at $0.75 \mathrm{~kg} \mathrm{ha}^{-1} \mathrm{fb}$ clodinafoppropargyl + metsulfuron methyl $0.4 \mathrm{~kg} \mathrm{ha}{ }^{1}$ $\left(\mathrm{W}_{5}\right)$ (Table 4). Tiwari et al.(2016) also reported significantly higher number of shoots $\left(345 \mathrm{~m}^{2}\right)$ in plots treated with clodinafoppropargyl + metsulfuron methyl at $0.4 \mathrm{~kg} \mathrm{~g}$ ha $^{1}$.

No significant variation was witnessed among tillage practices regarding grain yield of wheat. While, it varied significantly among the weed management practices (Table 4). 
Weed free $\left(\mathrm{W}_{7}\right)$ treatment registered the highest grain yield which was statistically at par with pendimethalin at $0.75 \mathrm{~kg} \mathrm{ha}^{-1} f b$ straw mulching at $4.0 \mathrm{t} \mathrm{ha}^{-1}\left(\mathrm{~W}_{4}\right)$, pendimethalin at $0.75 \mathrm{~kg} \mathrm{ha}^{-1} \mathrm{fb}$ clodinafoppropargyl + metsulfuron methyl $0.4 \mathrm{~kg} \mathrm{ha}^{1}$ $\left(\mathrm{W}_{5}\right)$ and straw mulching $f b$ clodinafoppropargyl + MSM at $0.40 \mathrm{~kg} \mathrm{ha}^{-1}\left(\mathrm{~W}_{6}\right)$. Integrated use of pre and post emergence herbicide significantly reduced the density and dry weight of weed and increased grain yield of wheat as also reported by Kumar et al. (2017) and Rana et al. (2017). Rahaman and Mukherjee (2009) also found maximum grain yield of wheat with pre-emergence application of pendimethalin and hand hoeing.

The highest straw yield was recorded under the treatment straw mulching $f b$ clodinafoppropargyl + MSM at $0.40 \mathrm{~kg} \mathrm{ha}^{-1}\left(\mathrm{~W}_{6}\right)$ which was statistically at par with weed free $\left(\mathrm{W}_{7}\right)$, pendimethalin at $0.75 \mathrm{~kg} \mathrm{ha}^{-1} f b$ straw mulching at $4.0 \mathrm{t} \mathrm{ha}^{-1}\left(\mathrm{~W}_{4}\right)$ and pendimethalin at $0.75 \mathrm{~kg} \mathrm{ha}{ }^{-1} f b$ clodinafop-propargyl + metsulfuron methyl $0.4 \mathrm{~kg} \mathrm{ha}^{1}\left(\mathrm{~W}_{5}\right)$ (Table 4). Straw yield was increased with the application of rice straw $\left(6 \mathrm{t} \mathrm{ha}^{-1}\right)$, compared to the control (Bharat et al., 2012).

Zero tillage fetched significantly the highest return rupee ${ }^{-1}$ invested (2.06) than conventional tillage (1.45) (Table 4). Among the weed management practices, pendimethalin at $0.75 \mathrm{~kg} \mathrm{ha}^{-1} \mathrm{fb}$ clodinafoppropargyl + metsulfuron methyl $0.4 \mathrm{~kg} \mathrm{ha}^{1}$ $\left(\mathrm{W}_{5}\right)$ achieved the highest return rupee $^{-1}$ invested (2.07), followed by pendimethalin at $0.75 \mathrm{~kg} \mathrm{ha}^{-1}\left(\mathrm{~W}_{2}\right)(2.03)$ and pendimethalin at $0.75 \mathrm{~kg} \mathrm{ha}^{-1} \mathrm{fb}$ straw mulching at $4.0 \mathrm{t} \mathrm{ha}^{-1}$ $\left(\mathrm{W}_{4}\right)$ (1.87) (Table 4). Integrated use of pendimethalin at $0.75 \mathrm{~kg}_{\mathrm{ha}}^{-1} f b$ straw mulching at $4.0 \mathrm{t} \mathrm{ha}^{-1}\left(\mathrm{~W}_{4}\right)$ and pendimethalin at $0.75 \mathrm{~kg} \mathrm{ha}{ }^{-1} f b$ clodinafop-propargyl + metsulfuron methyl $0.4 \mathrm{~kg} \mathrm{ha}{ }^{1}\left(\mathrm{~W}_{5}\right)$ recorded the highest gross return, net return as well as return per rupee invested over remaining treatments in wheat. This was due to higher grain and straw yield of wheat obtained from the above treatments and less cost of cultivation. Similar monetary benefit was also reported by Singh et al. (2004) and Singh (2014).

It can be concluded that zero tillage along with integrated use of straw mulching at $4.0 \mathrm{t}$ $\mathrm{ha}^{-1}$ with pre-emergence herbicide pendimethalin at $0.75 \mathrm{~kg} \mathrm{ha}^{-1}$ or post emergence application of ready mix herbicide clodinafop-propargyl + metsulfuron-methyl at $0.4 \mathrm{~kg}^{-1} \mathrm{ha}^{-1}$ or integrated use of preemergence herbicide pendimethalin at $0.75 \mathrm{~kg}$ $\mathrm{ha}^{-1}$ with post emergence application of ready mix herbicide clodinafop-propargyl + metsulfuron-methyl at $0.4 \mathrm{~kg}^{-1}$ appeared to be promising for effective and economic weed management in wheat.

\section{References}

Baghestani, M.A, Zand, E., Soufizadeh, S., Beheshtian, M., Haghighi, A., Barjasteh, A., Birgani, D.G. and Deihimfard, R. 2008. Study on the efficacy of weed control in wheat (Triticumaestivum L.) with tank mixtures of grass herbicides with broad-leaved herbicides. Crop Protection 27: 104-111.

Bharat, R., Kachroo, D., Sharma, R., Gupta, M. and Sharma, A.K. 2012. Effect of different herbicides on growth and yield performance of wheat. Indian Journal of weed Science44(2): 106-109.

Kumar, R., Singh, R.S. Jaidev and Kumar M.2017. Effect of herbicides on weeds, grain yield and soil health in wheat. Indian Journal of Weed Science 49(1): 88-89.

Kumar, V., Brainard,D.C., Sharma, P.C., Jat, H.S., Malik, R.K., Sharma, D.K. and McDonald, A. 2015. Conservation agriculture (CA) based practices reduced weed problem in wheat and caused shifts 
in weed seed bank community in ricewheat cropping systems. p. 142. In: Weed Science for Sustainable Agriculture, Environment, and Biodiversity. (Eds. Rao AN andYaduraju NT), Proceedings of 25th Asian Pacific Weed Science Society Conference, Hyderabad, India. October 13-16, 2015.

Mondal, S. and Duary, B. 2009. Effect of tillage and weed management practices on weed growth and productivity of rice-wheat cropping system in the Lateritic belt of West Bengal. In Souvenir of National Symposium on Agriculture in the Paradigm of Intergenerational Equity, 22-23 May, 2009, NS09-04-238, p. 44.

Pawar, J., Singh, R., Neelam, Kabdal, P., Prabhakar, D. and Kumar,S. 2017. Optimization rate of pinoxaden + clodinafop-propargyl for weed control in wheat. Indian Journal of Weed Science 49(2): 136-138.

Prasad, S., Singh, Y., Singh, R.P. and Singh, G. 2005. Effect of crop establishment weed control method and time of nitrogen application on late sown wheat. Indian Journal of Weed Science 37(1 \& 2): 9395.

Rahaman, S. and Mukherjee, P.K. 2009. Effect of herbicides on weed-crop association in wheat. Journal of Crop and Weed 5(2): 113-116.

Rana, S.S., Badiyala, D. and Brari, P. 2017. Evaluation of herbicide combinations for controlling complex weed flora in wheat. Indian Journal of Weed Science 49(4): 335-340.

Sharma, N., Thakur, N., Chopra, P., Kumar, S. and Badiyala, D. 2015. Evaluation of metsulfuron-methyl and clodinafop alone and in combination with other herbicides against weeds in wheat (Triticum aestivum L.) Research on Crops 16(3): 447-455.

Singh, A., Brar, S.S. and Virk, H.K. 2005. Acceleration of zero-tillage technology through farmer'sparticipatory approach in Punjab. Haryana Journal of Agronomy, 21(1): 37-40.

Singh, G., Singh, V.P. and Singh, M. 2004. Effect of Affinity on wheat and associated weeds. Indian Journal of Weed Science36(1\&2): 28-30.

Singh, R.K. 2014. Yield performance of zerotill wheat with herbicides in rice-wheat cropping system. Indian Journal of Weed Science 46(2): 174-175

Sirazuddin, Singh, S.P., Singh, V.P., Mahapatra, B.S. and Verma, H. 2016. Effect of weed control measures on yield, weed control, economics, energetics and soil microflora under different establishment methods of Wheat (Triticum aestivum L.). International Journal of Bio resource and Stress Management6(6): 736-743.

Susha, V.S., Das, T.K., Sharma, A.R. and Nath, C.P. 2014. Carry-over effect of weed management practices of maize on weed dynamics and productivity of succeeding zero and conventional till wheat. Ind. J. Agro. 59(1):41-47.

Tiwari, A., Verma, B.K., Tiwari, A. and Sharma, J.D. 2016. Effect on growth, yield and economics of wheat (Triticum aestivum L.) as affected by different herbicides. Research in Environment and Life Sciences9(4): 490-492.

\section{How to cite this article:}

Pratikshya Rani, S., B. Duary and Sidhartha Priyatam. 2020. Effect of Tillage and Weed management practices on Weed Control and Yield in Wheat. Int.J.Curr.Microbiol.App.Sci. 9(06): 2328-2335. doi: https://doi.org/10.20546/ijcmas.2020.906.285 